\title{
Paramagnetic Centres in Coal Macerals
}

\author{
G.P. SŁowiK And A.B. WięCKOWski* \\ Institute of Physics, Faculty of Physics and Astronomy, University of Zielona Góra \\ Szafrana 4a, 65-516 Zielona Góra, Poland
}

\begin{abstract}
The electron paramagnetic resonance (EPR) study of coal macerals: exinite, vitrinite and inertinite separated from clarain of the Polish medium-rank coal with carbon content of $85.6 \mathrm{wt} \% \mathrm{C}$, were performed. Using the continuous microwave saturation technique (measurements at $223 \mathrm{~K}$ and $291 \mathrm{~K}$ ), as a result of numerical analysis of recorded EPR spectra of exinite, vitrinite and inertinite, their multi-component structure was shown. The EPR spectra of exinite and vitrinite consist of three component lines: one Gaussian $G$ and two Lorentzians: $L 1$ and $L 3$. The EPR spectra of inertinite consist of two narrow lines with Lorentzian line shape $L 2$ and $L 3$. Temperature measurements of studied coal macerals in the range 100-373 K were done. It was evidenced that, in group of paramagnetic centres responsible for Lorentzian $L 1$ component line, paramagnetic centres with thermally excited triplet states $(S=1)$ exist. Existence of paramagnetic centres with thermally excited triplet states was not observed in inertinite.
\end{abstract}

PACS numbers: 61.66.Hq, 76.30.Rn, 89.30.ag

\section{Introduction}

The existence of anomalous temperature dependent paramagnetism for pairs of paramagnetic centres with spin $S=1$ was evidenced for the first time by Bleaney and Bowers [1]. For substances containing carbon, excited triplet states with spin $S=1$, were found by Yen et al. [2] in petroleum asphaltenes and by Yen and Young [3] in bitumens. In next years efforts were undertaken for finding thermally excited triplet states in coal [4] and in coal macerals heated in temperatures $300^{\circ} \mathrm{C}$ and $650{ }^{\circ} \mathrm{C}[5]$. The existence of paramagnetic centres (isolated aromatic radical clusters) in synthetic carbohydrate chars with excited multiplet states have been suggested by Smirnova et al. [6, 7]. The pulse electron paramagnetic resonance (EPR) spectroscopy was used by Ikoma et al. [8] to elucidate radical structures in carbonaceous solids of Argonne premium coals. Also the organic matter of carbonaceous meteorites contains paramagnetic centres characterised by a thermally accessible triplet state with spin $S=1[9,10]$.

Coal macerals are basic petrographic components of coal distinguished under microscope by reflectance and colour. The aim of this paper was evidence of complex, multi-component structure of EPR spectra for studied non-heated coal macerals: exinite, vitrinite and inertinite and the evidence of the existence in these samples of paramagnetic centres in thermally excited triplet states $(S=1)$.

\section{Experimental \\ 2.1. Materials}

The samples of exinite, vitrinite and inertinite were characterized by high purity, 75, 90 and 96 vol.\%, re-

* corresponding author; e-mail: Andrzej.Wieckowski@vp.pl spectively. The samples of coal macerals were obtained by centrifugation of the clarain in toluene-carbon tetrachloride mixtures. Clarain (common with vitrain, durain and fusain) is one of lithotype of bituminous coal. The samples of exinite, vitrinite and inertinite were placed in thin-wall glass tubes and to avoid interactions between molecules of atmospheric oxygen and paramagnetic centres present in studied macerals were evacuated $\left(10^{-4}\right.$ Torr $)$ and sealed.

\subsection{Measurements}

This work was performed by using for EPR measurements an X-band spectrometer with microwave frequency $9.4 \mathrm{GHz}$. The magnetic field modulation had the value $100 \mathrm{kHz}$. Maximum value of microwave power generated by klystron was $70 \mathrm{~mW}$. To avoid microwave saturation during EPR measurements with the continuous microwave saturation method of studied coal macerals, the used microwave power was approximately $0.7 \mathrm{~mW}$. EPR measurements with varied temperature were performed respectively in temperature ranges: 173-373 K (exinite), 133-373 K (vitrinite) and 123-293 K (inertinite).

\subsection{Fitting process of EPR spectra}

Recorded EPR spectra of studied non-heated coal macerals: exinite, vitrinite and inertinite were analysed numerically by two independent mathematical programs: Origin and Peak Fit. As a criterion of the quality of fitting, the theoretical function in the form of superposition of the first derivative functions of Gaussian $G$ and Lorentzian $L$ line shape with the experimental function given by the recorded EPR spectra of coal macerals the coefficient $\chi^{2}$ in the least-squares method was used. The EPR spectra of studied macerals: exinite, vitrinite and inertinite were fitted by 14 different combinations of above-mentioned functions. In Figs. 1a-3b 9 different 


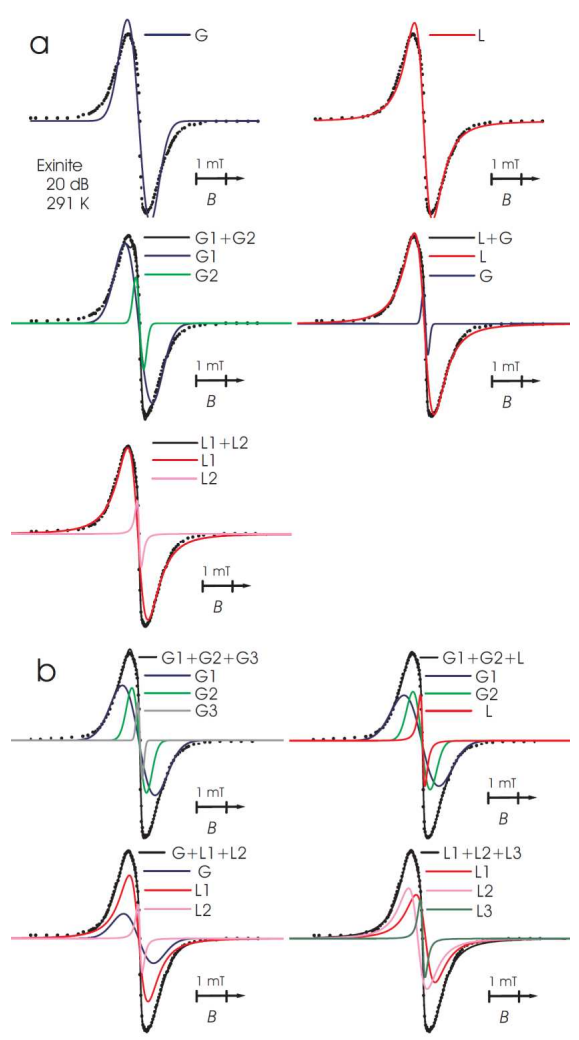

Fig. 1. (a) Fitting of the experimental EPR spectrum of exinite $(20 \mathrm{~dB}, 291 \mathrm{~K})$ as superposition of one or two derivatives of Gaussian and Lorentzian function. (b) Fitting of the experimental EPR spectrum of exinite $(20 \mathrm{~dB}, 291 \mathrm{~K})$ as superposition of three derivatives of Gaussian and Lorentzian function.

fittings were shown (20 dB, $291 \mathrm{~K})$, among them: one, two and three components for every examined by EPR non-heated maceral. The best fittings were characterized by the smallest value of this coefficient. The number of executed iterations in the process of fitting depends on the use of the mathematical program amounted from 40 to 200. The initial ordering of the functions used in the fitting process had no influence on the received values of the coefficient $\chi^{2}$, and by no means has it determined the quality of the obtained fitting.

\section{Results and discussion}

\subsection{Multicomponent structure of EPR spectra of macerals}

It was ascertained that the EPR spectra of studied exinite and vitrinite consists of three components two broad component lines having the shape of Gaussian curve $G$ and the shape of Lorentzian curve $L 1$, respectively, and one component line having the shape of Lorentzian curve L3. The words "broad", "narrow" relate to mutual relations between the linewidths $\Delta B_{\mathrm{pp}}$ of the component lines in the recorded EPR spectra of
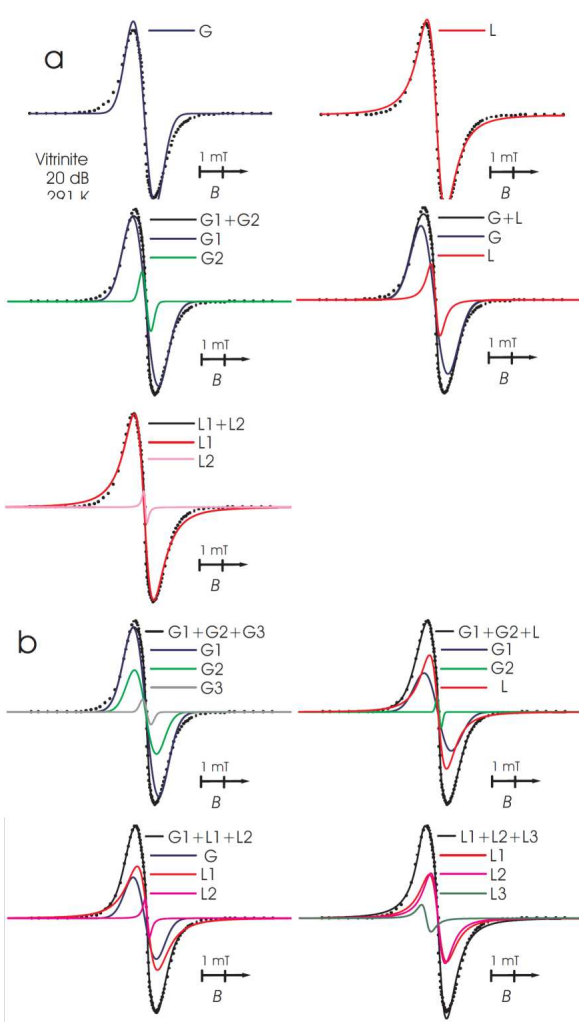

Fig. 2. (a) Fitting of the experimental EPR spectrum of vitrinite $(20 \mathrm{~dB}, 291 \mathrm{~K})$ as superposition of one or two derivatives of Gaussian and Lorentzian function. (b) Fitting of the experimental EPR spectrum of vitrinite $(20 \mathrm{~dB}, 291 \mathrm{~K})$ as superposition of three derivatives of Gaussian and Lorentzian function.

coal macerals. In the EPR spectra of exinite and vitrinite broad lines appear, having the Gaussian $G$ and Lorentzian $L 1$ line shape with the values: $G(0.95 \mathrm{mT}$; $291 \mathrm{~K} ; 20 \mathrm{~dB})$ and $L 1(0.59 \mathrm{mT} ; 291 \mathrm{~K} ; 20 \mathrm{~dB})$ for exinite, and $G(0.88 \mathrm{mT} ; 291 \mathrm{~K} ; 20 \mathrm{~dB})$ and $L 1(0.59 \mathrm{mT} ; 291 \mathrm{~K}$; $20 \mathrm{~dB}$ ) for vitrinite. In EPR spectra of two coal macerals mentioned above, also narrow lines with Lorentzian L3 line shape $(0.14 \mathrm{mT} ; 291 \mathrm{~K} ; 20 \mathrm{~dB})$ - exinite and $(0.12 \mathrm{mT} ; 291 \mathrm{~K} ; 20 \mathrm{~dB})$ - vitrinite, are present. The EPR spectra of inertinite consist of two narrow lines with Lorentzian line shape $L 2:(0.32 \mathrm{mT} ; 291 \mathrm{~K} ; 20 \mathrm{~dB})$ and L3 (0.12 mT; $291 \mathrm{~K} ; 20 \mathrm{~dB})$.

\subsection{Microwave saturation of EPR spectra of macerals}

Analyzing the changes of individual parameters of component lines (linewidth $\Delta B_{\mathrm{pp}}$, integral intensity $I$ ) recorded during measurements by use of the continuous microwave saturation technique, it was ascertained that the character of changes are similar in both temperatures of measurements, $223 \mathrm{~K}$ and $291 \mathrm{~K}$ (Fig. 4). On this base we can conclude that in the studied samples of coal macerals the dominant key role plays the spin-spin relaxation between paramagnetic centres. 

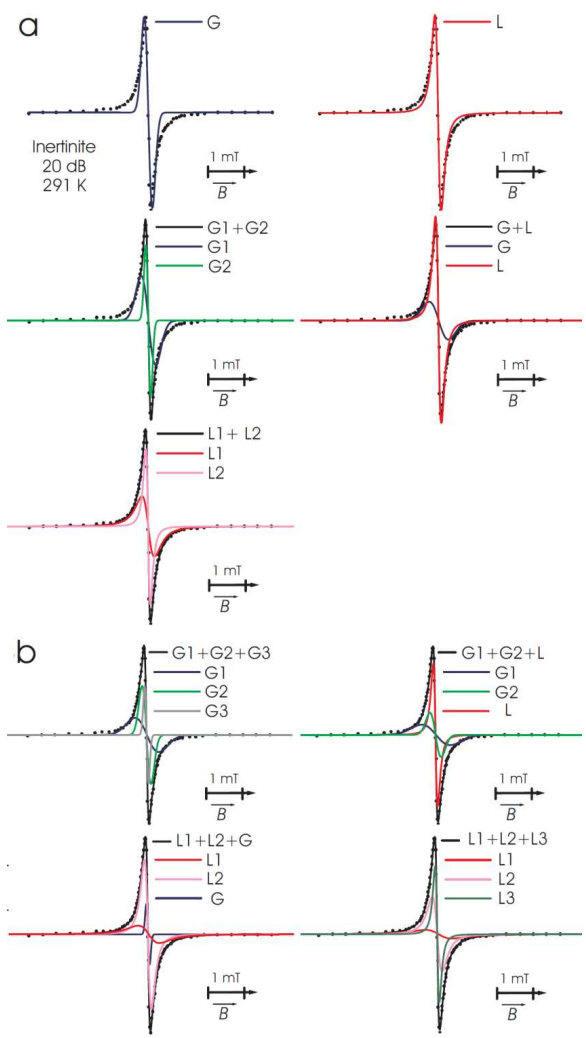

Fig. 3. (a) Fitting of the experimental EPR spectrum of inertinite $(20 \mathrm{~dB}, 291 \mathrm{~K})$ as superposition of one or two derivatives of Gaussian and Lorentzian function. (b) Fitting of the experimental EPR spectrum of inertinite $(20 \mathrm{~dB}, 291 \mathrm{~K})$ as superposition of three derivatives of Gaussian and Lorentzian function.

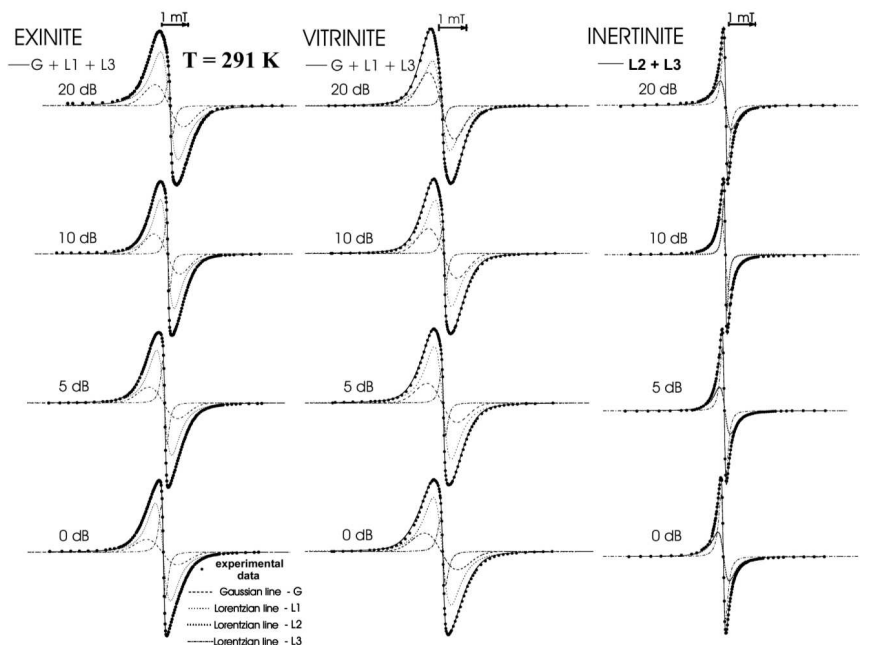

Fig. 4. Influence of microwave power on the components of EPR spectrum for exinite, vitrinite and inertinite in the range $0-20 \mathrm{~dB}$.
Individual component lines (Gaussian $G$ line shape, Lorentzian $L 1$ line shape, Lorentzian $L 2$ line shape and Lorentzian L3 line shape) in recorded EPR spectra of studied coal macerals: exinite, vitrinite and inertinite saturate in different, characteristic for given line way, which determined changes of line shape in the process of the continuous microwave saturation. It is also stated that in obtained EPR spectra the broad component lines $(G, L 1)$ saturate at lower values of microwave power than appropriate narrow component lines [11, 12].

It can be concluded that paramagnetic centres belonging to aromatic structures containing a relatively small number of aromatic rings or cyclic aliphatic compounds generate broad component lines. Paramagnetic centres of multi-ring aromatic structures generate the narrow component lines $(L 2, L 3)[13]$.

\subsection{Excited triplet states $(S=1)$}

As a result of thermal EPR study carried out in the temperature range 100-373 K (Fig. 5a,b), it was ascertained [14] that paramagnetic centres responsible for arising all component lines in EPR spectra of studied coal macerals in the form of Gaussian and Lorentzian line shape (except component $L 1$ ), exist in doublet ground state $(S=1 / 2)$ and fulfil the Curie law in the form

$$
I=\frac{C}{T} \text {. }
$$

Only in the group of paramagnetic centres responsible for component lines with Lorentzian line shape $L 1$ in EPR spectra of exinite and vitrinite some part of cen-

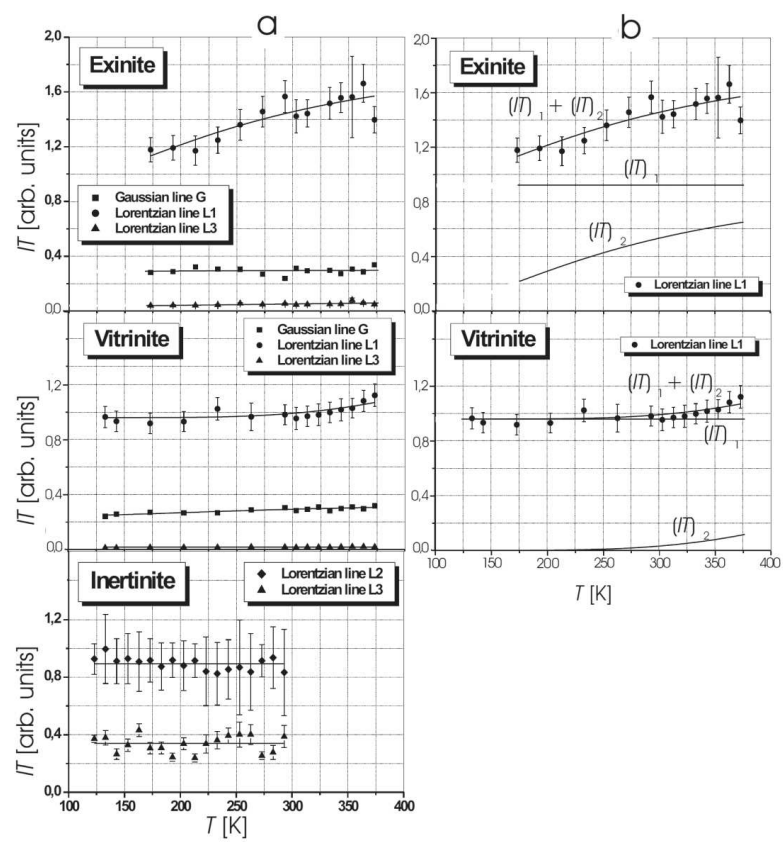

Fig. 5. (a) Intensity-temperature product (IT) versus temperature $T$ for the EPR components of exinite, vitrinite, and inertinite. (b) Intensity-temperature product (IT) versus temperature $T$ for Lorentzian $L 1$ line of exinite and vitrinite. 
tres (about 10-40\%; $373 \mathrm{~K}$ ) occur, which exist in excited triplet state $(S=1)$. These centres do not fulfil the Curie law and the generated by them dependence of the intensity of the EPR component line $L 1$ can be described by the formula given by Bleaney and Bowers [1]:

$$
I=\frac{1}{T}\left[C+\frac{B}{3+\exp (J / k T)}\right] .
$$

However papers exist (e.g. [15]), where recorded EPR spectra of coal were treated as single lines, without performing their numerical analysis. In result, the authors did not ascertain the existence of paramagnetic centres in excited triplet state $(S=1)$ in studied coals and possible deviations from the Curie law $(I=C / T)$ attributing to measured systematic errors.

In comparison to the papers presented above [2-10, 15] dealing with the existence of thermally excited triplet state $(S=1)$ in carbonaceous materials, our results prove that excited triplet states are also present in paramagnetic centres in non-heated coal macerals: exinite and vitrinite. These paramagnetic centres are giving the component EPR line of Lorentzian $L 1$ line shape.

\section{Conclusions}

On the base of performed EPR study of non-heated coal macerals (by the continuous microwave saturation method and temperature measurements): exinite, vitrinite and inertinite obtained from clarain of Polish medium-rank we ascertained that:

1. The EPR spectra studied coal macerals: exinite, vitrinite and inertinite characterized complex multi-component structure. Recorded EPR spectra of exinite and vitrinite consist of three components: Gaussian and Lorentzian lines $(G, L 1, L 3)$ and EPR spectra of inertinite consist of two component Lorentzian lines $(L 2, L 3)$.

2. Values of microwave power of saturation of individual component lines in EPR spectra of exinite, vitrinite and inertinite is different for broad $(G, L 1)$ and narrow $(L 2, L 3)$ components in recorded EPR spectra.

3. Spin-spin relaxation is dominant for paramagnetic centres present in examined exinite, vitrinite and inertinite.

4. All paramagnetic centres present in studied coal macerals exist in doublet ground state $(S=1 / 2)$ and only some part of centres (about 10-40\%;
$373 \mathrm{~K}$ ) having the broad Lorentzian $L 1$ line shape exist in excited thermally triplet state $(S=1)$.

5. Paramagnetic centres in excited triplet state $(S=1)$ were not found in inertinite.

\section{Acknowledgments}

We thank Professor Dr. Barbara Pilawa (Medical University, Sosnowiec) for the maceral samples and M.Sc. Wanda Wojtowicz (University of Zielona Góra) for her assistance in the EPR measurements.

\section{References}

[1] B. Bleaney, K.D. Bowers, Proc. R. Soc. Lond., Series A 214, 451 (1952).

[2] T.F. Yen, J.G. Erdman, A.J. Saraceno, Anal. Chem. 34, 694 (1962).

[3] T.F. Yen, D.K. Young, Carbon 11, 33 (1973).

[4] S. Duber, A.B. Więckowski, Fuel 63, 1474 (1984).

[5] A.B. Więckowski, B. Pilawa, L. Świątkowska, W. Wojtowicz, G.P. Słowik, M. Lewandowski, J. Magn. Reson. 145, 62 (2000).

[6] T.I. Smirnova, A.I. Smirnov, R.B. Clarkson, R.L. Belford, Solid State Commun. 91, 319 (1994).

[7] T.I. Smirnova, A.I. Smirnov, R.B. Clarkson, R.L. Belford, J. Phys. Chem. 98, 2464 (1994).

[8] T. Ikoma, O. Ito, S. Tero-Kubota, Energy Fuels 16, 40 (2002).

[9] D. Gourier, L. Binet, A. Skrzypczak, S. Derenne, F. Robert, Spectrochim. Acta A Mol. Biomol. Spectrosc. 60, 1349 (2004).

[10] O. Delpoux, D. Gourier, L. Binet, H. Vezin, S. Derenne, F. Robert, Spectrochim. Acta A Mol. Biomol. Spectrosc. 69, 1301 (2008).

[11] G.P. Słowik, A.B. Więckowski, Mol. Phys. Rep. 34, 146 (2001).

[12] G.P. Słowik, A.B. Więckowski, Appl. Magn. Reson. 24, 437 (2003).

[13] O. Ito, H. Seki, M. Ino, Bull. Chem. Soc. Jpn. 60, 2967 (1987).

[14] G.P. Słowik, W. Wojtowicz, A.B. Więckowski, Acta Phys. Pol. A 108, 171 (2005).

[15] K.S. Rothenberger, R.F. Sprecher, S.M. Castellano, H.L. Retcofsky, in: Magnetic Resonance of Carbonaceous Solids, Eds. R.E. Botto, S. Sanada, Symp. of 1989 Int. Chemical Congress of Pacific Basin Societes, Honolulu (Hawaii) 1989, Advances in Chemistry Series, Vol. 229, American Chemical Society, Washington DC 1993, Ch. 26, p. 581. 